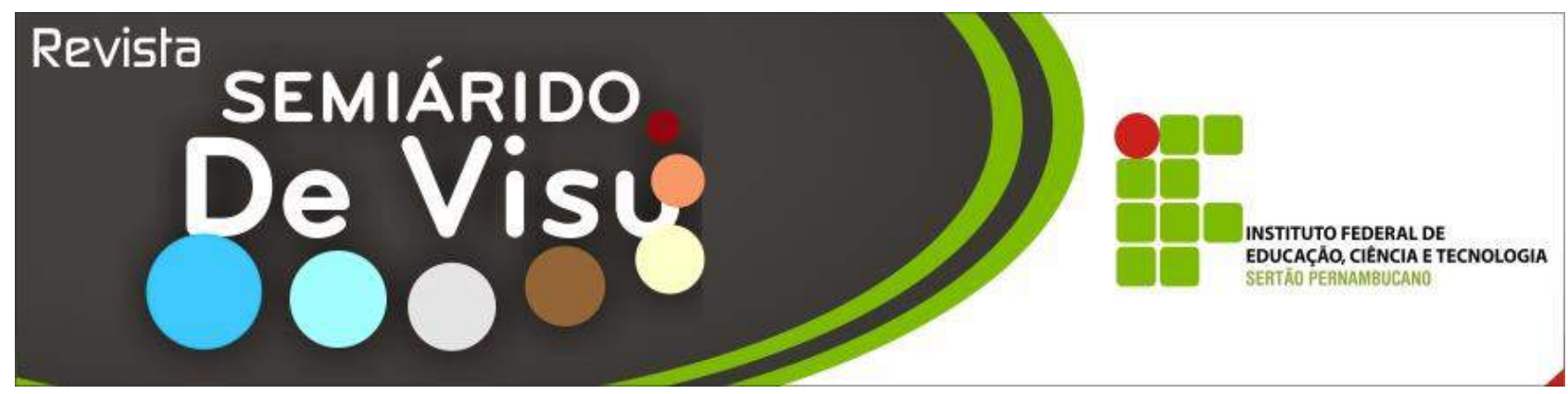

\title{
Efeitos de diferentes níveis de desfolha sobre a fisiologia, a produção e a qualidade das uvas e dos vinhos da variedade Syrah em condições tropicais semiáridas brasileiras
}

\author{
Mariana Barros Almeida ${ }^{1}$; Elizabeth Orika Ono ${ }^{2}$ \\ ${ }^{1,2}$ Instituto Federal de Educação Ciência e Tecnologia do Sertão Pernambucano - Campus Petrolina Zona Rural - Rodovia BR 235, \\ Km 22, Projeto Senador Nilo Coelho - N4, CEP: 56300-000 | Petrolina/PE - Brasil, Fone: (87) 2101-8050 - E-mail: \\ marib_almeida@yahoo.com.br ${ }^{1}$;
}

\begin{abstract}
RESUMO: A realização da desfolha de plantas de videira é uma prática bastante utilizada em países de clima temperado para modificar a incidência solar e melhorar as características enológicas de uvas e vinhos. Em condições tropicais ainda não se têm dados dessa prática, assim, o presente trabalho teve como objetivo avaliar o efeito de diferentes níveis de desfolha sobre a fisiologia, a produção e a qualidade de uvas da variedade Syrah em condições tropicais semiáridas. O estudo foi realizado na Fazenda Ouro Verde em Casa Nova, Bahia. Os tratamentos consistiram na prática de 5 níveis de desfolha nos ramos no início da fase de cacho fechado. Da desfolha a colheita foram avaliados: taxa de fotossíntese e produção das plantas; teores de sólidos solúveis, acidez titulável, $\mathrm{pH}$, polifenóis totais, taninos e antocianinas, bem como seu potencial enológico. O delineamento experimental foi em blocos casualizados com 4 repetições sendo 5 plantas por tratamento, totalizando 100 plantas no experimento. Pode-se constatar que de acordo com o manejo empregado, dentre os parâmetros estudados apenas o teor de fenóis totais e antocianinas nas uvas e nos vinhos, bem como os dados biométricos dos cachos, tiveram diferença significativa entre os tratamentos, enquanto que os demais parâmetros estudados não sofreram interferência da desfolha.
\end{abstract}

Palavras-chaves: regiões áridas, vitis vinifera L., uva, vinho.

\section{Effects of different levels of defoliation on the physiology, production and quality of grapes and wines, variety Syrah, in brazilian tropical semiarid conditions}

\begin{abstract}
The defoliation of grapevine plants is widely used in temperate countries to modify the solar incidence and improve the oenological characteristics of grapes and wines. In tropical conditions there is not data on this practice, so this study aimed to evaluate the effect of different levels of defoliation on the physiology, production and quality of Syrah grapes in semi-arid tropical conditions. The study was conducted at Ouro Verde Farm in Casa Nova, Bahia State. The treatments consisted of five levels of defoliation on the branches in early stage L of the Baggiolini scale. During the growing season and at harvest, the fruits were evaluated on rate of photosynthesis, plants production, fruit as soluble solids, titratable acidity, $\mathrm{pH}$, polyphenols, tannins and anthocyanins, as well as its oenological potential. The experimental design was randomized blocks with four replications and five plants per treatment, totaling 100 plants in the experiment. It was noted that according to crop management, among the parameters studied, only the content of total phenols and anthocyanins in grapes and wines, as well as the biometric data of the clusters had significant difference between treatments, while the other parameters did not suffer interference from defoliation.
\end{abstract}

Keywords: regions arid, vitis vinifera L., grape, wine. 


\section{Introdução}

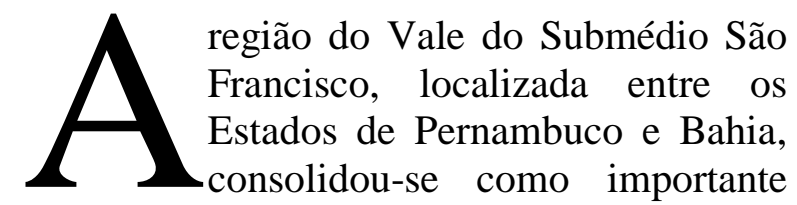
produtora de uvas finas de mesa. No entanto, nos últimos anos tem se verificado um crescimento expressivo do cultivo de uvas para vinho. Destaca-se como a principal região vitivinícola de clima tropical do mundo. Trata-se de uma região nova, com muitos aspectos a serem conhecidos e/ou melhor, compreendidos. A região possui características climáticas que confere total diferenciação em relação à viticultura tradicional em todo o mundo e seu próprio potencial vitivinícola está para ser, em grande parte, desvendado (MIOLO, 2003), tendo como principal característica a possibilidade de vegetação da videira durante os 12 meses do ano, podendo as práticas de colheitas serem realizadas durante todo o ano, podendo chegar até 3 safras/ano.

No Submédio São Francisco a área plantada com videira tem se expandido significativamente nos últimos anos, devido à modernização da agricultura brasileira, principalmente, pela implantação de projetos de irrigação que promovem transformações na estrutura produtiva que favorecem a integração econômica dessa região ao mercado agroindustrial. Grande parte desse crescimento deve-se ao forte interesse de empresas nacionais e internacionais em implantar grandes fazendas de uvas para vinho, a fim de processá-las durante todo o ano no Vale.

A variedade Syrah é a principal uva tinta para vinho cultivada na região do Submédio São Francisco (ORLANDO et al., 2008). É uma variedade muito adaptada ao clima quente que, segundo Castro et al. (2006) demonstra bom desempenho agronômico quando enxertada sobre 1103P.

O clima, o solo, o material vegetal (espécies e variedades), o sistema de condução e as técnicas de manejo do vinhedo têm grande influência na maturação fenólica das uvas, interferindo no tipo e no teor de compostos fenólicos (MARASCHIN, 2003).

A poda verde é uma prática cultural que vem sendo utilizada para melhorar as condições do dossel vegetativo dos vinhedos visando favorecer a qualidade da uva. A desbrota, desponta e a desfolha são modalidades de poda verde que interferem diretamente no dossel vegetativo e podem contribuir para que se alcance equilíbrio entre as partes vegetativas e reprodutivas da videira (MANDELLI et al., 2008).

A desfolha é uma técnica amplamente utilizada e tem como objetivo minimizar problemas climáticos encontrados durante a maturação da uva, como a precipitação e a umidade relativa do ar elevadas. O manejo do dossel vegetativo permite maior insolação e aeração do vinhedo, favorecendo o microclima próximo às folhas e aos frutos e ao aumento da qualidade (MANFROI et al., 1997)

A luz e a temperatura têm influência determinante na composição e na maturação da uva. A falta de luz nos cachos afeta o tamanho da baga, o $\mathrm{pH}$ do mosto, o teor de açúcares totais e o metabolismo do ácido málico, aumentando sua concentração, também provoca diminuição no conteúdo de fenóis totais e antocianinas.

Trabalhos realizados em várias regiões vitícolas mostram que desfolhar ligeiramente a zona dos cachos em diversos estádios fenológicos (HUNTER et al., 1995) pode aumentar o teor de sólidos solúveis e diminuir a acidez, o pH e o potássio (REYNOLDS et al., 1996). Isto ocorre porque se eliminam as folhas velhas que sombream os cachos, que pouco ou nada contribuem para a síntese de açúcares (MAIN e MORRIS, 2004; MURISIER e FERRETTI, 2004; PONI et al., 2005).

Experimentos conduzidos em vários países, como França, Itália, Estados Unidos, África do Sul, Austrália, Nova Zelândia e Chile evidenciam que o manejo do microclima da copa é um meio efetivo de aumentar a qualidade do vinho. No que se refere à composição analítica dos vinhos, poucos parâmetros analisados mostraram 
diferenças significativas (FREESE, 1988; ILAND, 1988). No entanto, análises sensoriais demonstraram que vinhos elaborados de uvas em condições de microclima manejado apresentaram escores superiores e foram considerados de melhor qualidade (HUNTER e VISSER, 1991). A prática da desfolha poderia ser um instrumento enológico para obtenção de um vinho com tipificação e identidade, proporcionando modificações na composição química.

O estudo da prática da desfolha em videiras viníferas cultivadas na região semiárida do Submédio do Vale do São Francisco é fundamental para o conhecimento das respostas da planta nessas condições, tanto em relação à produção da uva, quanto na qualidade do vinho. Assim, o presente trabalho teve como objetivo avaliar o efeito de diferentes níveis de desfolha sobre a fisiologia, a produção e a qualidade das uvas e dos vinhos da variedade Syrah em condições tropicais semiáridas.

\section{Material e Métodos}

\section{Matéria-prima}

O trabalho foi realizado no primeiro semestre de 2010, em vinhedo comercial, na Fazenda Ouro Verde Ltda, localizada na BR 235, km 40, Casa Nova (BA), Brasil. As plantas utilizadas foram da cultivar Syrah clone 100 com cerca de 5 anos e enxertadas no porta-enxerto IAC 766. As plantas foram conduzidas em sistema do tipo espaldeira, espaçadas de $2,5 \times 1,5 \mathrm{~m}$ sob irrigação por gotejamento de 4 litros por hora planta ${ }^{-1}$.

Após a determinação do ponto ideal de colheita, as uvas foram colhidas e encaminhadas ao laboratório de Enologia da Embrapa Semiárido, Petrolina-PE, onde foram vinificadas, através do método tradicional de Peynaud, (1997) de acordo com a figura abaixo.

Figura 1. Fluxograma de operações para produção de vinho tinto.

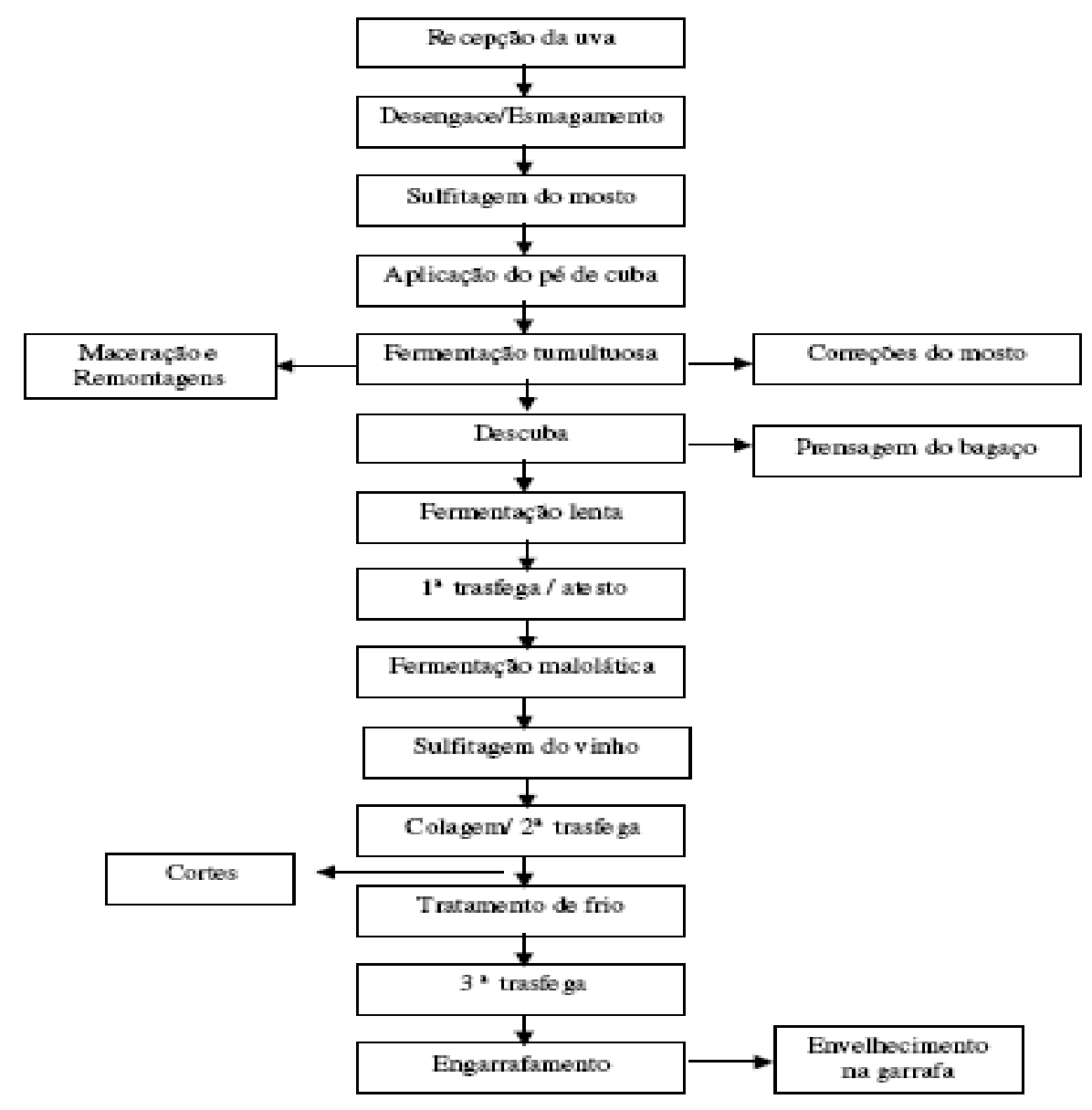




\section{Estádio fenológico}

O estádio fenológico da videira foi determinado, semanalmente, pelo método de observação visual, tomando como referência a escala de Baggiolini, divulgada em 1952 (BAILLOD e BAGGLIOLINI, 1993). Quando se considerou haver alguma heterogeneidade, em que era difícil a definição de apenas um estádio, procedeu-se a atribuição de duas letras.

\section{Desfolha}

Os diferentes níveis de desfolha foram realizados manualmente do lado leste da espaldeira, lado este que recebe menor incidência solar, em linhas instaladas no sentido NORTE- SUL, no início do estádio de cacho fechado (Estádio L) de acordo com a escala de Baggiolini, onde foram eliminadas folhas da região próxima aos cachos (até 40 $\mathrm{cm}$ acima do primeiro arame) conforme a figura 2. Os tratamentos consistiram na eliminação de: $\mathrm{T} 1=0$ folhas (testemunha); $\mathrm{T} 2=3$ a 4 folhas; $\mathrm{T} 3=5$ a 6 folhas; $\mathrm{T} 4=7$ a 8 folhas e $\mathrm{T} 5=9$ a 10 folhas, em ramos previamente marcados.

Figura 2. Fio de produção no sistema de condução espaldeira na qual foram realizadas as desfolhas até $40 \mathrm{~cm}$ acima do fio.

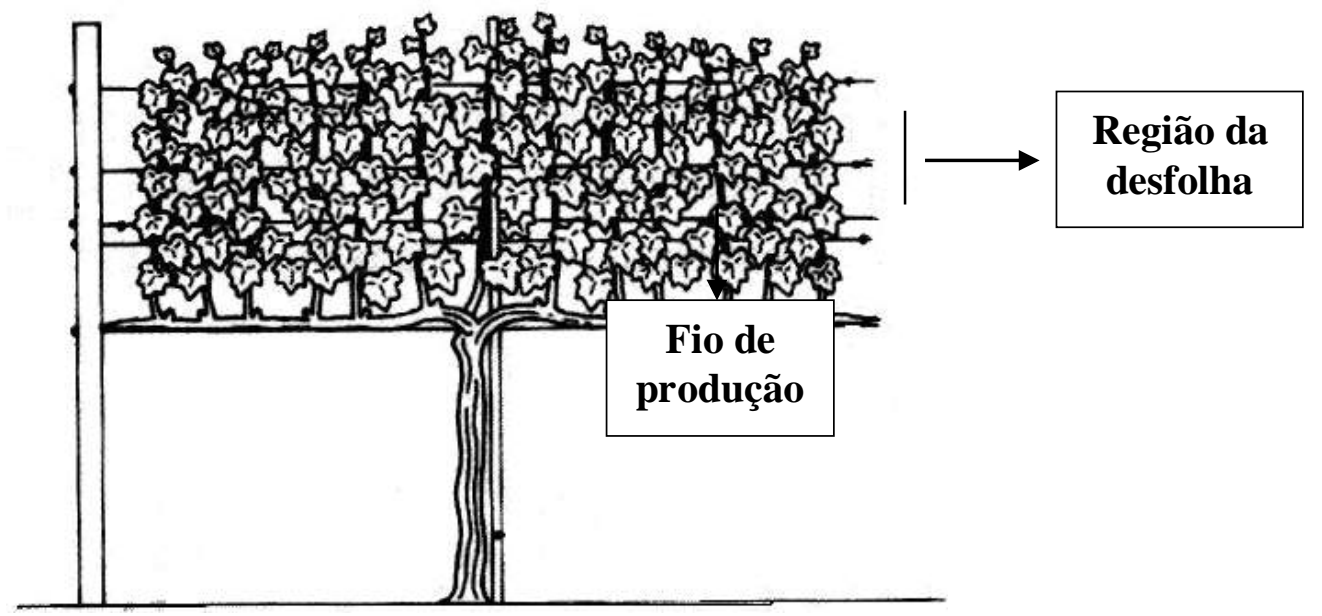

\section{Medidas de Trocas Gasosas}

Aos 48, 56, 62, 72 e 112 dias após a poda foram realizadas medidas de trocas gasosas utilizando o aparelho portátil de fotossíntese, Infra Red Gas Analyzer (IRGA, Li-6200, Licor Ltda., Lincoln, NE). As medidas de trocas gasosas foram realizadas no período da manhã, entre 9:00 e 11:00 horas, onde foram obtidos os valores de taxa de assimilação de $\mathrm{CO}_{2}\left(\mu \mathrm{mol} \mathrm{CO}_{2} \mathrm{~m}^{-2} \mathrm{~s}^{-1}\right)$

\section{Análises da Uva}

Os parâmetros referentes às características das bagas e cachos, bem como a composição física química dos mesmos, foram realizados após a colheita, aproximadamente 112 dias após a poda. As amostras representativas de cada parcela, constituídas de 60 cachos, foram pesadas em balança analítica marca Gibertini, em unidades de gramas, e medidas de comprimento e largura foram realizadas com o auxílio de um paquímetro manual e as medidas foram dadas através de leitura direta em $\mathrm{cm}$.

Os parâmetros físico- químicos, teor de sólidos solúveis (SS), composição fenólica (CF), acidez titulável (AT) e pH foram determinados de acordo com a metodologia descrita por Peynaud, (1997).

\section{Análises dos vinhos}

Após 30 dias de estabilização em garrafas, foram realizadas, em triplicata, as seguintes analises: $\mathrm{pH}$, Acidez total titulável, Teor de álcool, Acidez volátil, $\mathrm{SO}_{2} \mathrm{Livre}, \mathrm{SO}_{2}$ Total, Densidade, Extrato Seco, Açúcares 
Redutores, Antocianinas segundo a metodologia descrita por Peynaud, (1997).

\section{Delineamento experimental e análise estatística}

Para as análises de campo o delineamento experimental utilizado foi blocos inteiramente casualizados e para as análises de laboratório inteiramente casualizado em triplicata.

Os resultados obtidos foram submetidos à análise de variância sendo as médias comparadas pelo teste Tukey a $5 \%$ de significância com o auxílio do programa estatístico SAS 9.0 (Statistical Analysis System).

\section{Resultado e discussão}

\section{Estádios fenológicos}

No período onde foi realizado o trabalho (primeiro semestre de 2010), as precipitações que ocorreram estiveram dentro das médias encontradas em anos anteriores, com exceções dos meses de março, onde a média de precipitação é de $148 \mathrm{~mm}$ e nesse ano foi de $91 \mathrm{~mm}$ e no mês de Abril, onde a média é de $82 \mathrm{~mm}$ e ocorreu uma precipitação em torno de $148,3 \mathrm{~mm}$, porém, essa diferença de precipitação não interferiu na execução nem nos resultados do trabalho. Já os demais dados climático (radiação, insolação, temperatura e umidade relativa do ar) estiveram todos dentro das médias mensais.

Constatou-se que a duração dos ciclos fenológicos da videira no primeiro semestre de 2010 foi de 112 dias entre o intervalo da poda, realizada em março de 2010 à colheita realizada em junho 2010 (Tabela 1). Moura et al. (2009) estudando o ciclo de produção de dois anos da variedade Syrah no Vale do submédio São Francisco verificaram que a duração do ciclo fenológico é da ordem de $118 \pm 14$ dias. Amorim et al. (2005), trabalhando com a mesma cultivar no município de Três Corações, região sul de Minas Gerais, constataram que o ciclo de produção foi de 164 dias. Na Austrália, Ginaster et al. (1998), citados por Amorim et al. (2005), relataram que a uva cv. Syrah precisou de 193 dias para completar seu ciclo produtivo em clima temperado.

Tabela 1. Duração dos sub-períodos fenológicos em um ciclo de produção ( $1^{\circ}$ semestre de 2010$)$ da videira cv. Syrah, Casa Nova-BA (PO-BR- Poda a brotação; BR-FL- Brotação a floração; FL-MA- Floração a maturação; MA-CL Maturação a colheita; PO-CO- Poda a colheita)

Sub-períodos fenológicos

\begin{tabular}{lccccr} 
Ano. Semestre & PO-BR & BR - FL & FL - MA & MA - CL & PO - CO \\
\hline $2010 / 1^{\circ}$ & 8 & 20 & 43 & 40 & 112 \\
\hline
\end{tabular}

Favero et al. (2008) em trabalho realizado para a cultivar Syrah nos anos de 2005 e 2006 no sul de Minas Gerais verificaram que a duração do ciclo foi de 195 dias e 180 dias, respectivamente.

Souza et al. (2010) verificaram para a cultivar Syrah, cultivada em ciclo de verão no município de Caldas (MG), foi colhida aos 154 dias, ou seja, em torno de 33 dias a menos do que o verificado nos trabalhos anteriormente citados.
Desta forma, no caso da vitivinicultura do Vale do Submédio São Francisco, é possível programar datas de poda, quebra de dormência, aplicação de defensivos, colheita e também otimizar o emprego da mão de obra nas diversas fases do ciclo, reduzindo os tratos fitossanitários, resultando em economia de insumos (SOUZA LEÃO e SOARES., 2010). Além disso, em função do histórico do regime de chuvas de uma região subtropical, pode-se programar a poda de frutificação e quebra de dormência no verão para a 
obtenção da produção fora de época, em que a maturação dos cachos ocorre em condições climáticas mais favoráveis, uma vez que, neste período, é importante que não haja excesso de umidade nos cachos, evitando-se a ocorrência de podridões fúngicas que prejudicam $\mathrm{o}$ processo fermentativo $\mathrm{da}$ vinificação (JUBILEU et al., 2010).

\section{Taxa de Assimilação de $\mathrm{CO}_{2}$}

A atividade fotossintética das folhas, que alcançou o valor máximo de 11,5 $\mu \mathrm{mol}$ $\mathrm{CO}_{2} \mathrm{~m}^{-2} \mathrm{~s}^{-1}$ no início da maturação, reduziu-se lenta e progressivamente até o final do ciclo, situando-se próximo a 9,0 $\mu \mathrm{mol} \mathrm{CO}_{2} \mathrm{~m}^{-2} \mathrm{~s}^{-1}$, principalmente, nos tratamentos com desfolha de até 6 folhas (Tt3), valores que podem ser considerados satisfatórios para a videira, conforme observado na Alemanha ( DURING, 1992), Austrália (DOWNTOWN et al., 1987) e Portugal (CASTRO et al., 1993).

Para as desfolhas mais drásticas (T4 e T5) não foi observada redução da taxa fotossintética e sim, sua manutenção durante o período de estudo. Assim, pode-se dizer que a desfolha nesse caso, não alterou a produção de fotoassimilados.

Ao decidir a época e intensidade da desfolha, deve ser levada em consideração a idade das folhas (VASCONCELOS e CASTAGNOLI, 2000), a capacidade fotossintética das folhas removidas e daquelas que permanecem na planta. Poni et al. (1994) observaram que a fotossíntese alcançava o máximo de assimilação aos 30-35 dias de idade das folhas, o que corresponde ao momento em que as folhas atingem $o$ tamanho máximo. Castro et al. (2006) defendem que a partir de certa idade, a contribuição da folha para a produção de fotossimilados vai diminuindo gradualmente, até ser praticamente nula na maturação.

Até o estádio fenológico de baga ervilha, são as folhas basais adultas que contribuem com a maioria dos fotossimilados para o desenvolvimento das bagas. Nas fases posteriores do ciclo, são as folhas dos terços médio e superior que se manifestam funcionalmente ativas com maior importância na produção de fotoassimilados e acumulação de constituintes dos cachos (HUNTER et al., 1994).

Zufferey e Murisier (2000) concluíram que o perfil de evolução da atividade fotossintética das folhas apresentava valor máximo na floração e no estabelecimento das bagas; redução até o cacho fechado; estável ao longo da fase de pintor e redução durante a maturação das bagas. $\mathrm{Na}$ fase final do ciclo são as folhas dos ramos netos que mais contribuem para o potencial fotossintético da planta, dado que nessa fase as folhas estão mais ativas (CASTRO et al., 2005, 2006).

Ao efetuar a desfolha, cerca de um mês após a plena floração, a produção de assimilados já não é afetada, pois outras folhas adultas que se desenvolvem encontramn-se em plena atividade fotossintética (VASCONCELOS e CASTAGNOLI, 2000). Castro e Lopes (1990) consideram que a desfolha, se realizada durante a maturação, com a remoção das folhas situadas abaixo do cacho poderá ser positiva e Castro e Cruz (2001) consideram a época do pintor e vindima como adequada para a realização da desfolha.

\section{Análises biométricas}

Em relação à massa de 60 bagas houve diferença significativa entre os tratamentos. As bagas da testemunha (T1) e do tratamento com maior intensidade de desfolha (T5) apresentaram as menores massas, 103,8 $\mathrm{g}$ e $107,5 \mathrm{~g}$, respectivamente (Tabela 2), enquanto que bagas das desfolhas intermediárias (T2, T3 e T4) apresentaram bagas mais pesadas com valores semelhantes (119,1, 120,8 e $123,1 \mathrm{~g}$, respectivamente). Pode-se constatar que os diferentes níveis de desfolha interferiram na massa das bagas, porém, vale ressaltar que em se tratando de uvas de vinho é importante se ter uma boa relação polpa e semente para que seja possível obter uvas de qualidade para elaboração de vinhos finos, portanto nem sempre as maiores bagas são desejadas. 
A massa e comprimento dos cachos sofrerarm influência em relação aos níveis de desfolha empregados (Tabela 2). A desfolha de 5 a 6 folhas (T3) promoveu a formação de cachos mais pesados $(159,4 \mathrm{~g})$ e mais longos $(12,1 \mathrm{~cm})$, enquanto que os tratamentos sem desfolha (T1), com desfolha de 3 a 4 folhas
(T2) e de 9 a 10 folhas (T5) foram semelhantes entre si, apresentando cachos de menores massas e mais curtos. Vale ressaltar que em se tratando de uvas para vinho, o que se busca é a qualidade e maturação dos compostos presentes nas bagas e cachos, não sendo importante a dimensão dos cachos.

Tabela 2 Massa de 60 bagas (g), massa dos cachos (g), largura e comprimento dos cachos de videira 'Syrah' submetidos a diferentes níveis de desfolha.

\begin{tabular}{lcccc}
\hline Tratamentos & $\begin{array}{c}\text { Massa } \\
\text { 60 bagas }(\mathrm{g})\end{array}$ & $\begin{array}{c}\text { Massa } \\
\text { cachos }(\mathrm{g})\end{array}$ & $\begin{array}{c}\text { Largura } \\
\text { cachos }(\mathrm{cm})\end{array}$ & $\begin{array}{c}\text { Comprimento } \\
\text { cachos }(\mathrm{cm})\end{array}$ \\
\hline Testemunha (T1) & $103,8 \mathrm{~b}$ & $139,2 \mathrm{c}$ & $4,9 \mathrm{a}$ & $10,9 \mathrm{~b}$ \\
3 a 4 folhas (T2) & $119,1 \mathrm{a}$ & $144,6 \mathrm{c}$ & $4,7 \mathrm{a}$ & $11,4 \mathrm{~b}$ \\
5 a 6 folhas (T3) & $120,8 \mathrm{a}$ & $159,4 \mathrm{a}$ & $4,8 \mathrm{a}$ & $12,1 \mathrm{a}$ \\
7 a 8 folhas (T4) & $123,1 \mathrm{a}$ & $154,4 \mathrm{~b}$ & $4,9 \mathrm{a}$ & $11,8 \mathrm{ab}$ \\
9 a 10 folhas (T5) & $107,5 \mathrm{~b}$ & $147,2 \mathrm{c}$ & $4,8 \mathrm{a}$ & $11,4 \mathrm{~b}$ \\
\hline
\end{tabular}

Médias seguidas de mesma letra nas colunas, não diferem significativamente entre si pelo teste Tukey a $5 \%$ de probabilidade.

\section{Análises bioquímicas}

Na tabela 3 observa-se que não houve diferença significativa entre os tratamentos quanto ao teor de sólidos solúveis e acidez titulável do mosto de bagas da cv 'Syrah', permanecendo os valores em torno de $21,0^{\circ}$ Brix e 60,0 meq $\mathrm{L}^{-1}$, respectivamente.
Trabalhos realizados por Lavezzi et al. (1994) mostraram que não há relação entre intensidade de desfolha e a concentração de sólidos solúveis. Os resultados podem expressar uma adaptação das videiras quanto à acumulação de açúcares, independente dos níveis de desfolha.

Tabela 3. Teor de sólidos solúveis (SS), acidez titulável (AT) em mosto de bagas de videira 'Syrah' submetidos a diferentes níveis de desfolha.

\begin{tabular}{ccc}
\hline Tratamentos & $\begin{array}{c}\text { SS } \\
\left({ }^{\mathrm{o}} \mathrm{Brix}\right)\end{array}$ & $\begin{array}{c}\text { AT } \\
\left(\mathrm{meq} / \mathrm{L}^{-1}\right)\end{array}$ \\
\hline Testemunha (T1) & $21,2 \mathrm{a}$ & $62,7 \mathrm{a}$ \\
3 a 4 folhas (T2) & $21,8 \mathrm{a}$ & $60,0 \mathrm{a}$ \\
5 a 6 folhas (T3) & $21,0 \mathrm{a}$ & $60,7 \mathrm{a}$ \\
7 a 8 folhas (T4) & $21,7 \mathrm{a}$ & $57,7 \mathrm{a}$ \\
9 a 10 folhas (T5) & $21,8 \mathrm{a}$ & $61,0 \mathrm{a}$ \\
\hline
\end{tabular}

Médias seguidas de mesma letra nas colunas, não diferem significativamente entre si pelo teste Tukey a $5 \%$ de probabilidade.

Diversos estudos relacionados à desfolha constataram redução nas concentrações de sólidos solúveis nas bagas (KLIEWER et al., 1967), particularmente se a desfolha for muito intensa (ZOECKLEIN et al., 1998). Esta redução poderá estar relacionada com a redução das folhas fotossinteticamente ativas, ou com o fato da área foliar remanescente ser insuficiente para a completa maturação das bagas. Pelo contrário, outros autores defendem que a desfolha provoca o aumento da concentração de açúcares na baga (REYNOLDS et al., 1986b; VASCONCELOS e CASTAGNOLI, 2000). Este aumento no teor em açúcares deve-se à perda de água nas bagas que, mais expostos devido à desfolha, vêem aumentadas 
as taxas de respiração e evaporação, aumentando a conce

ntração de sólidos solúveis (ZOECKLEIN et al., 1992). Esta variação pode também ser atribuída ao aumento da temperatura das bagas, o qual induz ao aumento da taxa de translocação de fotoassimilados (REYNOLDS et al., 1986). Mas, vários estudos mostram ausência de efeitos da desfolha no teor de açúcares (BLEDSOE et al., 1988; CASTRO et al., 2007b).

Van Leeuwen et al. (2004) admitem que $88 \%$ da variação de açúcar da videira dependem, principalmente, da cultivar, do solo e do ano climático decorrente, isso pode justificar a infinidade de trabalhos com resultados tão antagônicos. Também segundo Manfroi et al. (2006), a concentração e as substâncias que compõem os frutos são influenciadas por vários fatores, como a maturação, a cultivar, a época do ano, as práticas culturais e as condições climáticas.

Para obtenção de vinhos de qualidade as uvas devem apresentar boas características quanto à sua composição, como a quantidade de açúcares, acidez titulável, teor de antocianinas e polifenóis totais, porém vale ressaltar que não uma regra de composição ideal, deve-se haver um equilíbrio entre os diferentes compostos.
A qualidade interna do fruto depende, em grande parte, da radiação solar recebida durante o período de crescimento vegetativo (INTRIERI e FILIPPETTI, 2000), condição esta que no Semiárido Tropical do Vale do São Francisco ocorre em grande intensidade na maior parte do ano.

\section{Composição fenólica da uva}

O teor de antocianinas totais das películas das bagas de uva 'Syrah' apresentou diferença estatística entre os tratamentos (Tabela 4), onde a maior concentrações desses compostos pode ser encontrada na desfolha de 3 a 4 folhas (T2), enquanto que a testemunha (T1) e os tratamentos com maior desfolha apresentaram teores menores de compostos fenólicos. Assim, pode-se sugerir que uma leve desfolha pode ser positiva para o aumento da concentração desses compostos, importantes para a elaboração de vinhos tintos.

De acordo com estudos realizados em outras variedades de Vitis vinifera, por Flanzy et al. (1972), as tintas apresentam conteúdo médio de antocianinas de $2295 \mathrm{mg} \mathrm{kg}^{-1}$ de uva, encontrando-se entre 95 a $98 \%$ na película e os restantes $2 \%$ no engaço.

Tabela 4. Teor de antocianinas totais (Ant, mg $100 \mathrm{~g}^{-1}$ ) em bagas de videira 'Syrah' submetidos a diferentes níveis de desfolha.

\begin{tabular}{cc}
\hline Tratamentos & $\begin{array}{c}\text { Ant } \\
\left(\mathrm{mg} \mathrm{100} \mathrm{g} \mathrm{g}^{-1}\right)\end{array}$ \\
\hline Testemunha (T1) & $115 \mathrm{c}$ \\
3 a 4 folhas (T2) & $138,5 \mathrm{a}$ \\
5 a 6 folhas (T3) & $120,1 \mathrm{~b}$ \\
7 a 8 folhas (T4) & $126,7 \mathrm{~b}$ \\
9 a 10 folhas (T5) & $122,2 \mathrm{~b}$ \\
\hline Médias seguidas de mesma letra nas colunas não diferem
\end{tabular}

Médias seguidas de mesma letra nas colunas não diferem

Significativamente entre si pelo teste Tukey a $5 \%$ de probabilidade.

A forma como os pigmentos antociânicos evoluem ao longo da maturação é bastante influenciada por um conjunto de fatores (tipo de cultivo, condições ambientais, práticas culturais, regime hídrico entre outros). A luz e a temperatura são os fatores climáticos mais importantes na biossíntese das antocianinas. Pirie e Mullins (1977) verificaram que em videiras que receberam $15 \%$ da luz da testemunha, havia redução de $60 \%$ no teor em antocianinas durante a primeira semana de coloração da baga. $\mathrm{O}$ autor citado considera que a luz aumenta o teor de açúcares da película e que este induz a 
acumulação das antocianinas (LAUREANO, 1988).

A evolução do conteúdo antociânico caracteriza-se, geralmente, em três fases: na primeira, o nível antociânico apresenta ligeiro incremento, na segunda, caracteriza-se por incremento mais acentuado no nível de antocianinas e por último, na terceira fase, observa-se estabilização seguida de decréscimo até $o$ final da maturação tecnológica (RIBÉREAU-GAYON, 1982; JORDÃO et al., 1998a).

Em variedades de Vitis vinifera $\mathrm{L}$. as antocianinas são produzidas durante o período de maturação na fase do pintor. Esta fase é caracterizada pela mudança de cor e textura da baga devido à acumulação de antocianinas nas películas das uvas tintas.

Um período de maturação prolongado provoca redução do nível antociânico, associado ao murchamento das bagas (SOMERS, 1976; PIRIE e MULLINS, 1977).

As práticas culturais que aumentam a exposição dos cachos ao sol, geralmente, provocam aumento dos compostos fenólicos e da densidade da cor dos vinhos (MAZZA et al., 1999).

Durante o presente trabalho, as temperaturas médias ficaram em torno de 24,6 mínima em junho a 28,1 máxima em fevereiro, alguns autores consideram que para algumas variedades, as temperaturas ótimas para a acumulação de antocianinas nas bagas se situam entre os $15-25^{\circ} \mathrm{C}$ durante o dia e 10$20^{\circ} \mathrm{C}$ durante a noite (KLIEWER, 1970). Sugere-se que temperaturas acima de $35^{\circ} \mathrm{C}$ ou a baixo de $15^{\circ} \mathrm{C}$, bem como grandes amplitudes térmicas entre o dia e a noite, fazem reduzir a acumulação de antocianinas (MATEUS et al., 2002). Além disso, a deficiência ou o excesso de humidade e de irrigação tendem a diminuir o conteúdo antociânico nas uvas (JORDÃO et al., 1998b).

$\mathrm{Na}$ análise dos valores de antocianinas e polifenóis totais nas películas das uvas 'Cabernet Sauvignon' observa-se que a prática da desfolha ocasionou aumento significativo do teor desses compostos, comprovando que a maior irradiação solar nos cachos ocasiona maior síntese dessas substâncias, o que está de acordo com Morrison e Noble (1990), Disegna et al. (2005) e Pereira et al. (2005).

A prática da desfolha também proporcionou a elaboração de vinhos com maior intensidade de cor, assim como, maior teor de antocianinas totais, principalmente, no tratamento com leve desfolha de 3 a 4 folhas (T2) (Tabela 4).

A acumulação de compostos fenólicos depende de uma adequada densidade de folhagem, uma boa exposição solar e uma temperatura moderada ao nível dos cachos, fatores controlados pela desfolha (CHAMPAGNOL, 1984). Muitos outros autores referem, igualmente, o efeito positivo da desfolha na composição fenólica do mosto.

Outros, no entanto, não verificaram qualquer influência da desfolha no teor de antocianinas e fenóis totais, bem como na intensidade corante do mosto (ZOECKLEIN et al., 1992). Já Dokoozlian e Kliewer (1996) fazem notar que temperaturas muito elevadas durante a maturação inibem a formação de antocianinas e fenóis totais. Smart (1987) concluiu que a exposição direta dos cachos, além de aumentar a temperatura, é favorável à síntese de antocianinas e compostos fenólicos totais.

Botelho et al. (2007), no já referido ensaio com a casta 'Alfrocheiro', obtiveram resultados diferentes em função do ano do ensaio. Em 2005, a desfolha não provocou qualquer diferença significativa no teor de antocianinas totais, fenóis totais e intensidade corante; enquanto que, em 2006, as antocianinas totais e a intensidade corante foram significativamente superiores na planta com desfolha.

\section{Análises Bioquímicas e composição fenólica dos vinhos}

Os resultados obtidos permitiram
verificar que não houve diferença
significativa $(\mathrm{p}<5 \%)$ entre os tratamentos para
as variáveis densidade, $\mathrm{pH}$ e açúcares
redutores. No entanto, os níveis de desfolha
apresentaram influência significativa $(\mathrm{p}<5 \%)$


sobre as variáveis; teor alcoólico, acidez titulável e volátil, dióxido de enxofre livre e total, antocianinas totais e compostos fenólicos totais (Tabela 5).

Segundo Rizzon e Miele (2003), a densidade do vinho é consequiência da graduação alcoólica e da quantidade de açúcar residual presentes. O açúcar presente na uva será transformado em álcool pelas leveduras, durante a fermentação alcoólica (DELANOE et al., 1987 apud BLASI, 2004). No entanto, a uva contém ainda uma pequena quantidade de açúcares, não-fermentescíveis, cerca de $1 \mathrm{gL}^{-1}$, e que se encontram no vinho.

$\mathrm{O}$ conhecimento do $\mathrm{pH}$ se torna de suma importância, pois através dele pode se avaliar a resistência do vinho à infecção bacteriana ou tendência a casse férrica (BLASSI 2004) ou porcentagem de $\mathrm{SO}_{2}$ presente na forma livre. Para Rizzon e Miele (2002), o pH interfere na cor e exerce um efeito pronunciado sobre o gosto, além de contribuir para uma boa fermentação (PEYNAUD, 1997).

O teor alcoólico mais elevado foi obtido nos vinhos do tratamento 2 . Todos os tratamentos apresentaram-se dentro do permitido pela legislação, a qual estabelece o valor mínimo de $10^{\circ} \mathrm{GL}$ e máximo de $14^{\circ} \mathrm{GL}$. $\mathrm{O}$ álcool etílico possui características antisépticas, pois impede o desenvolvimento de agentes patogênicos no vinho (SILVA, 1999).

Os teores de $\mathrm{SO}_{2}$ livre, os quais variaram entre 20,9 e 27,3, resultaram em uma acidez total (Tabela 5), de acordo com o permitido pela legislação de $55-130 \mathrm{meq} \mathrm{L}^{-1}$, e acidez volátil equilibrada com teores entre 5,0 e 5,5 meq $\mathrm{L}^{-1}$ sendo que a lei permite no máximo $20 \mathrm{meq} \mathrm{L}^{-1}$. A acidez volátil está diretamente relacionada com a concentração de $\mathrm{SO}_{2}$, pois provavelmente teores de $\mathrm{SO}_{2}$ adequados inibem o desenvolvimento das bactérias responsáveis pela produção de ácido acético (PEYNAUD, 1997).

Dessa forma, para todos os tratamentos de desfolha $\mathrm{O} \mathrm{SO}_{2}$ livre e total apresentaram-se de acordo com o recomendado por Peynaud (1997) e RibéreauGayon (2004) que é de 20-30 $\mathrm{mg} \mathrm{L}^{-1}$ de $\mathrm{SO}_{2}$ livre para vinhos tintos e valores de $\mathrm{SO}_{2}$ tota de $90-100 \mathrm{mg} \mathrm{L}^{-1}$ de $\mathrm{SO}_{2}$ combinado, onde o tratamento 2 apresentou as maiores concentrações de $\mathrm{SO}_{2}$ livre e total.

Os teores de extrato seco $(24,8-26,3 \mathrm{~g}$ $\mathrm{L}^{-1}$ ) estão diretamente relacionados com a concentração de açúcares presentes na uva, visto que os maiores teores de extrato seco foram encontrados para o tratamento 2 . Segundo Barnabé et al., (2007), vinhos com maior porcentagem de extrato seco apresentam na análise sensorial maior nota atribuída ao quesito corpo. Esta característica é devido às maiores quantidades de componentes da casca na composição do vinho.

Para a concentração de antocianinas totais no vinho, as maiores concentrações foram encontradas no tratamento com leve desfolha de 3 a 4 folhas (T2). As antocianinas são pigmentos naturais responsáveis por uma vasta gama de cores em vegetais, frutas e outros produtos derivados, tal como o vinho tinto. Antocianinas e flavonóis tem importância fundamental para estrutura química, equilíbrio gustativo e longevidade de vinhos tintos, sendo extraídos da uva na fase de maceração.

As diferentes técnicas de vinificação permitem a extração destas moléculas nos primeiros quatro a seis dias de maceração. A sua concentração nos vinhos tintos obtidos de uvas de castas de Vitis vinifera varia entre 350 $\mathrm{mg} \mathrm{L}^{-1}$ e $1100 \mathrm{mg} \mathrm{L}^{-1}$ (PEYNAUD, 1997).

Disegna et al. (2005) também constataram maior conteúdo de antocianinas em uvas e vinhos 'Tannat' provenientes de vinhedos com desfolha no Uruguai. Estudos realizados por Polenta (1996), em vinhos 'Cabernet Sauvignon' de Santana do Livramento (RS) e por Antes (2008), em vinhos 'Cabernet Sauvignon' de Bagé (RS) da safra 2007 demonstraram diferenças estatísticas entre si quanto ao teor de. antocianinas totais dos vinhos com os maiores valores no tratamento com desfolha.

As concentrações dos compostos fenólicos totais foram maiores para $\mathrm{O}$ tratamento 2 . O vinho tinto possui um grande número de constituintes polifenólicos, como flavonóides, estilbenos, antocianinas, ácidos 
fenólicos e taninos, que exercem efeitos sobre a saúde human.

A concentração desses compostos na uva e no vinho depende de fatores como a cultivar de videira, as condições de cultivo, estágio de maturação e os métodos de vinificação empregados. Além disso, a composição fenólica do vinho pode variar durante todo o processo de vinificação, estabilização e armazenamento do vinho devido às reações que ocorrem durante o envelhecimento, como as reações de condensação, polimerização, oxidação e precipitação.

Tabela 5. Análise química dos vinhos elaborados a partir de uvas cv. Syrah submetidas a diferentes manejos de desfolha do dossel vegetativo.

\begin{tabular}{|c|c|c|c|c|c|}
\hline \multirow[t]{2}{*}{ Variáveis } & \multicolumn{5}{|c|}{ Tratamentos } \\
\hline & $\mathbf{T 1}$ & $\mathbf{T} 2$ & T3 & $\mathbf{T 4}$ & T5 \\
\hline Densidade & $0,993 \mathrm{a}$ & $0,993 \mathrm{a}$ & $0,993 \mathrm{a}$ & $0,993 \mathrm{a}$ & $0,993 \mathrm{a}$ \\
\hline Teor alcoólico (\%) v/v & $13,2 \mathrm{~b}$ & $13,5 \mathrm{a}$ & $12,9 \mathrm{e}$ & $13,1 \mathrm{c}$ & $13,0 \mathrm{~d}$ \\
\hline $\mathrm{pH}$ & $3,5 \mathrm{a}$ & $3,5 \mathrm{a}$ & $3,5 \mathrm{a}$ & $3,5 \mathrm{a}$ & $3,5 \mathrm{a}$ \\
\hline Acidez Total $\left(\mathrm{meq} \mathrm{L}^{-1}\right)$ & $96 \mathrm{a}$ & $89 \mathrm{~d}$ & $91 \mathrm{c}$ & $87 \mathrm{e}$ & $93 \mathrm{~b}$ \\
\hline Acidez Volátil (meq L L) & $5,3 \mathrm{c}$ & $5,0 \mathrm{~d}$ & $5,5 \mathrm{a}$ & $5,3 \mathrm{c}$ & $5,4 \mathrm{~b}$ \\
\hline $\mathrm{SO}_{2}$ Total $\left(\mathrm{mg} \mathrm{L}^{-1}\right)$ & $69,1 \mathrm{~d}$ & 76,8 a & $58,9 \mathrm{e}$ & $75,9 \mathrm{~b}$ & $74,2 \mathrm{c}$ \\
\hline $\mathrm{SO}_{2}$ Livre $\left(\mathrm{mg} \mathrm{L}^{-1}\right)$ & $24,7 \mathrm{c}$ & $27,3 \mathrm{a}$ & 20,9 e & $27,3 \mathrm{a}$ & $23,0 \mathrm{~d}$ \\
\hline Extrato Seco $\left(\mathrm{g} \mathrm{L}^{-1}\right)$ & $25,0 \mathrm{~d}$ & $26,3 \mathrm{a}$ & $24,8 \mathrm{e}$ & $25,4 \mathrm{c}$ & $25,6 \mathrm{~b}$ \\
\hline Açúcares Redutores $\left(\mathrm{g} \mathrm{L}^{-1}\right)$ & $1,3 \mathrm{a}$ & $1,3 \mathrm{a}$ & $1,3 \mathrm{a}$ & $1,3 \mathrm{a}$ & $1,3 \mathrm{a}$ \\
\hline Antocianinas $\left(\mathrm{mg} \mathrm{L}^{-1}\right)$ & $549,4 \mathrm{e}$ & $774,7 \mathrm{a}$ & $589,8 \mathrm{~d}$ & $684,2 \mathrm{~b}$ & $616,9 \mathrm{c}$ \\
\hline
\end{tabular}

Médias seguidas de mesma letra nas colunas, não diferem significativamente entre si pelo teste Tukey a $5 \%$ de probabilidade.

\section{Conclusão}

Os diferentes níveis de desfolha empregados no presente trabalho não influenciaram significativamente o ciclo fenológico da videira que foi de aproximadamente 112 dias, ciclo esse considerado curto a vista de outras regiões, mas pelas condições climáticas diferenciadas, esta antecipação é normal para as condições do Vale. Para as taxas de assimilação de $\mathrm{CO}_{2}$ os tratamentos empregados não apresentaram redução da taxa fotossintética e sim, sua manutenção durante o período de estudo.

Pode-se constatar que os diferentes níveis de desfolha interferiram tanta nas massas dos cachos como nas bagas, onde o tratamento testemunha (T1) e o tratamento com maior intensidade de desfolha (T5) apresentaram as menores massas, enquanto que as bagas e os cachos das desfolhas intermediárias (T2, T3 e T4) apresentaram valores superiores. Porém os tratamentos empregados não interferiram nas variáveis tanto de comprimento dos cachos como de teor de sólidos solúveis e acidez titulável das bagas.

Os níveis de desfolha avaliados neste trabalho influenciaram significativamente a composição dos vinhos tropicais 'Syrah', no primeiro semestre de 2010, sendo o tratamento com leve desfolha de 3 a 4 folhas (T2), o tratamento que proporcionou maior qualidade no que se refere a composição desses vinhos, principalmente, em relação aos teores de antocianinas totais e compostos fenólicos totais, os quais foram os mais elevados, bem como teor alcoólico.

\section{Referências Bibliográficas}

AMORIM, D.A.; FAVERO, A.C.; REGINA, M.A. Produção extemporânea da videira, cultivar Syrah nas condições do sul de Minas Gerais. Revista Brasileira de Fruticultura, Jaboticabal, v.27, n.2, p.327-331, 2005. 
ANTES, S. Sobrematuração da uva na composição e qualidade de vinhos cv. Tannat e Cabernet Sauvignon da Região de Bagé RS. 2008. 69 f. Dissertação (Mestrado) - Curso de pós-graduação em Ciência e Tecnologia Agroindustrial, Universidade Federal de Pelotas, 2008.

BAILLOD, M.; BAGGIOLINI, M. Les stades repères de la vigne. Revue Suisse Vitic. Arboric Hortic., v.25, p.7-9, 1993.

BARNABÉ, D.; VENTURINI FILHO, W. G; BOLINI, H. M. A. Análise Descritiva Quantitativa de Vinhos Produzidos com Uvas Niágara Rosada e Bordô. Brazilian Journal of Food Technology, v. 10, n. 2, p. 122-129, abr./jun. 2007.

BLASI, T.C. Análise do consumo e constituintes químicos de vinhos produzidos na Quarta Colônia de Imigração Italiana do Rio Grande do Sul e sua relação com as frações lipídicas sangüíneas. Santa Maria, 2004. 91f. Dissertação de Mestrado.

BLEDSOE, A.M.; KLIEWER, W.M.; MAROIS, J.J. Effects of timing and severity of leaf removal on yield and fruit composition of Sauvignon Blanc grapevines. American Journal of Enology and Viticulture, Davis, v.39, n.1, p.49-54, 1988.

BOTELHO, M.; CRUZ, A.; RODRIGUES, C.; RODRIGUES, A.; GOMES, C.; CASTRO, R. Efeitos da densidade do cobertoe da monda no comportamento agronómico e ecofisiológico da casta Alfrocheiro. In: Simpósio de Viticultura do Alentejo, 7., 2007, Évora. Actas ... Évora, 2007. p.279-285.

CASTRO, R.; LOPES, C.; CLÍMACO, P.; CHAVES, M.M. Résultats écophysiologiques de Ia vigne au Portugal. Aspects hydriques. In: G.E.S.C.O. Compte rendu $\mathrm{n}^{\circ} 6$, 172-177, C.I.V.C. e Mumm Perrier-Jouet. Reims, 1993.

CASTRO, R.; CRUZ, A .; FIGUIERA, L.; MOREIRA, M.; RODRIGUES, C.; GOMES, C. Shoot density and leaf removal effects on microclimate, yield, fruit composition and wine quality of the Portuguese vine variety Touriga Nacional. Geisenheim, Comptes Rendus. XIV Gesco, v.2, p.705-711, 2005.

CASTRO, R.; CRUS, A.; BOTELHO, M. Tecnologia Vitícola. Ministério da Agricultura, Pesca e Florestas/ Direcção Geral de Agricultura da Beira Litoral/Comissão Vitivinícola da Bairrada, Coimbra, 2006. 160p.

CASTRO, R.; CRUZ, A.; MAGALHÃES, A.; GUIMARAENS, D.; SOUSA, M.; ABADE, E.; CUNHA, M.; QUEIRÓZ, J. Training of grapevines in narrow terraces: cv. Touriga Nacional. In: INTERNATIONAL SYMPOSIUM - GESCO. POREC, 15., 2007b, Croatia, Anais ... Croatia, p.1160-1166.

CASTRO, R.; CRUZ, A. Intervenções em verde na vinha. Crsopa, Série II, nº 27. 2001.

CASTRO, R.; LOPES, C. Influência do sistema de poda e de condução da vegetação sobre a fertilidade e produção na casta Fernão Pires. In: CONGRESSO IBÉRICO DE CIÊNCIAS HORTÍCOLAS, 1., 1990. Actas de Horticultura, 1990. p.314-319.

CHAMPAGNOL, F. Elements de physiologie de Ia vigne et de viticulture générale. Montpellier, 1984. 351p.

DISEGNA, E. et al. Avances en el estúdio del momento y intensidad del deshojado y su incidência em la produccion y calidad de uvas y vinos del cultivar 'Tannat'. In: X CONGRESSO LATINO-AMERICANO DE VITICULTURA E ENOLOGIA, 2005, Bento

DOKOOZLIAN, N.K.; KLIEWER, W.M. Influence of light on the grape berry growth and composition varies during fruit development. J. Am. Soc. Hort. Sci., v.121, p.869-874, 1996.

DURING, H. Gas exchange of grapevines leaves as affected by soil factors. In : INTERNATIONAL SYMPOSIUM ON GRAPEVINE PHYSIOLOGI, 4., 1992, San 
Michel All'adige Italie. Proceedings... San Michel, 1992.

DOWNTOWN, W.J.S.; GRANT, W.J.R.; LOVEYS, B.R. Diurnal changes in the photosynthesis of field-grown grape vines. New Phytologist, Cambridge, v.105, p.71-80, 1987.

FAVERO, A.C.; AMORIM, D.A.; MOTA, R.V.; SOARES, A.M.; REGINA, M.A. Viabilidade de produção da videira 'Syrah' em ciclo de outono inverno na região Sul de Minas Gerais. Revista Brasileira de Fruticultura, Jaboticabal, v.30, n.3, p.685-690, 2008.

FREESE, P.K. Canopy modification and fruit composition, Auckland. In: INTERNATIONAL SYMPOSIUM FOR COOL CLIMATE VITICULTURE AND OENOLOGIE, 2., 1988, Auckland. Proceedings ... Auckland, New Zealand Soe. Vitie. Oenol, p.134-6, 1988.

GINESTAR, C.; EASTHAM, J.; GRAY, S.; ILAND, P. Use of sap-flow sensors to schedule vineyard irrigation. II. Efects of post-veraison water deficits on composition of shiraz grapes. American Journal of Enology and Viticulture, Davis, v.49, n.4, p.421-428, 1998.

HUNTER, J.J.; RUFFNER, H.P.; VOLSCHENK, C.G. et al. Partial defoliation of Vitis vinifera L. cv. Cabernet Sauvignon/99 Richter: Effect on root growth, capony efficiency, grape composition, and wine quality. American Journal of Enolology and Viticulture, v.46, n.3, p.306-313, 1995.

HUNTER, J.J. et al. The effect of partial defoliation on quality characteristics of Vitis vinifera L. cv. Cabernet Sauvignon grapes. II. Skin sugar, and wine quality. Am. J. Enol. Vitic., Davis, v.42, n.1, p.13-18, 1991.

HUNTER, J.J.; VISSER, J.H. The effect of partial defoliation, leaf position and developmental stage of the vine on the photosynthetic activity of Vitis vinifera L. cv. Cabernet Sauvignon. S. Afr. J. Enol. Vitic., v.9, n.2, p.9-15, 1988.
ILAND, P.G. Leafremoval effects on fruit composition, Auckland, $1988 . \quad$ In: INTERNATIONAL SYMPOSIUM FOR COOL CLIMATE VITICULTURE AND OENOLOGIE 2., 1988, Auckland. Proceedings... Auckland, New Zealand Soe. Vitic. Oenol, 1988. p. 137-8.

INTRIERI, C.; FILIPPETTI, I. Innovations and outlook in grapevine training systems and mechanization in North-Central Italy. In: AMERICAN SOCIETY FOR ENOLOGY AND VITICULTURE ANNUAL MEETING, 50., 2000, Seattle, WA. Proceedings... Seattle: The American Society for Enology and Viticulture, 2000. p.170-184.

JORDÃO, A.M.; RICARDO DA SILVA, J.M.; LAUREANO, O. Influência da rega na composição fenólica das uvas tintas da casta Touriga Francesa (Vitis vinifera L.). Cienc. Tecnol. Aliment., v.2, n.2, p.60-73, 1998a.

JORDÃO A.M.; RICARDO-DA-SILVA J.M.; LAUREANO O. Evolution of anthocyanins during grape maturation of two varieties (Vitis vinfera L.), Castelão Frances and Touriga Francesa. Vitis, v.37, n.2, p.93-94, 1998 b.

JUBILEU, B.S. et al. Caracterização fenológica e produtiva das videiras 'Cabernet Sauvignon' e 'Alicante' (Vitis vinifera L.) produzidas fora de época no Norte do Paraná. Revista Brasileira de Fruticultura, Jaboticabal, v.32, n.2, p.451-462, 2010.

KLIEWER, W.M; LIDER, L.A.; SHULTZ, H.B. Influence of artificial shading of vineyards on the concentration of sugar and organic acids in grapes. American Journal of Enology and Viticulture, Davis, v.18, p.78-86, 1967b.

LAUREANO, O. A matéria corante dos vinhos tintos. Relações com a cor e a origem dos vinhos. 1988. Dissertação apresentada às provas de acesso à categoria de Investigador Auxiliar. Instituto Superior de Agronomia - UTL.

MAIN, G.L.; MORRIS, J. R. Leaf-removal effects on Cynthiana yield, juice composition, 
and wine composition. American Journal of Enology and Viticulture, Davis, v.55, n.2, p.147-152, 2004.

MANDELLI, F; MIELE, A. Uvas viníferas para processamento em regiões de clima temperado. EMPRESA BRASILEIRA DE PESQUISA AGROPECUÁRIA. EMBRAPA, 2003. Online. Acesso em: 02 mar. 2010. Disponível em: http://www.cnpuv.embrapa.br/publica/sprod/ UvasViniferasRegioesClimaTemperado/poda. htm\#desfolha

MANFROI, V. et al. Efeito de diferentes épocas de desfolha e de colheita na composição do vinho Cabernet Sauvignon. Ciência Rural, Santa Maria, v.27, n.1, p.139146, 1997.

MATEUS, N.; SILVA, M.A.S.; SANTOSBUELGA, C.; RIVAS-GONZALO, J.C.; DE FREITAS, V. Identification of anthocyaninflavonol pigments in red wines by NMR and mass spectrometry. J. Agric. Food Chem., v.50, p.2110-2116, 2002.

MAZZA, G.; FUKUMOTO, L.; DELAQUIS, P.; GIRARD, B.; EWERT, B. Anthocyanins, phenolics, and color of Cabernet Fanc, Merlot, and Pinot Noir wines from British Columbia. J. Agric. Food Chem., v.47, p.4009-4017, 1999.

MURISIER, F.; FERRETTI, M. Trial on leaf removal in the zone grape buch of Merlot grapevines in Ticino, Switzerland. Effects on the quality of grapes and wines. Revue Suisse de Viticulture, Arboriculture et Horticulture, Lausanne, v.36, n.6. p.355-359, 2004.

MANFROI, L.; MIELE, A.; RIZZON, L.A.; BARRADAS, C.I.N. Composição físicoquímica do vinho Cabernet Franc proveniente de videiras conduzidas no sistema lira aberta. Ciência e Tecnologia de Alimentos, v.26, n.2, p.290-296, 2006.
MARASCHIN, R.P. Caracterização química de vinhos Cabernet Sauvignon produzidos na Serra Gaúcha (Enfase em compostos fenólicos). 2003. Dissertação (Mestrado em Biotecnologia) - Centro de Ciências Biológicas da Universidade Federal de Santa Catarina, 2003.

MIOLO, A. Novas regiões: vinho de clima tropical. In: CONGRESSO BRASILEIRO DE VITICULTURA E ENOLOGIA, X, 2003. Anais...Bento Gonçalves: Embrapa Uva e Vinho/CYTED. p.141-144. 2003.

MOURA, M. S. B.; et al., Exigência Térmica e Caracterização Fenológica da Videira Cabernet Sauvignon no Vale São Francisco, Brasil. Disponível em: http://www.repdigital.cnptia.embrapa.br/bitstr eam /CPATSA /36771/ 1/OPB1654.pdf Acesso em: 15 março de 2009

MORRISON, J.C.; NOBLE, A.C. The effects of leaf and cluster shading on the composition of Cabernet Sauvignon grapes an on fruit and wine sensory properties. Am. J. Enol. Vitic., Davis, v.41, p.193-200, 1990.

ORLANDO, T.G.S.; PEDRO JÚNIOR, M.J.; SANTOS, A.O.; HERNADES, J.L.; SILVA, T. DAS G. Comportamento das cultivares Cabernet Sauvignon e Syrah em diferentes porta-enxertos. Ciênc. agrotec., Lavras, v.32, n.3, p.749-755, 2008.

PEREIRA, G.P. et al. Influência do microclima sobre o perfil metabólico de uvas Merlot cultivadas em Bordeaux-França. In: CONGRESSO LATINO-AMERICANO DE VITICULTURA E ENOLOGIA, 10., 2005, Bento Gonçalves, RS. Anais ... Bento Gonçalves: Embrapa, 2005. p.324.

PEYNAUD, E. Connaissance et travail du vin. Dunond: Paris, 1997, 340p.

PIRIE, A.; MULLINS, M.G. Interrelationships of sugars, anthocyanins, total phenols and dry weight in the skin of 
grape berries during ripening. Amer. J. Enol. Vitic., v.28, p.204-209, 1977.

POLENTA, G.A. Evolução dos compostos fenólicos durante a fermentação de mostos provenientes de três regiões do Rio Grande do Sul submetidos a diferentes tratamentos. 1996. 155f. Dissertação (Mestrado em Ciência e Tecnologia dos Alimentos) - Curso de pós-graduação em Ciência e Tecnologia dos Alimentos, Universidade Federal de Santa Maria, 1996.

PONI, S.; BERNIZZONI, F.; BRIOLA, G.; CENNI, A. Effects of early removal on cluster morphology, shoot efficiency and grape quality in two Vitis vinifera cultivars. Acta Horticulturae, The Hague, n.689, p.217-226, 2005.

PONI, S.; LAKSO, A.N.; TURNER, J.R.; MELIOUS, R.E. Interactions of crop level and late season water stress on growth and physiology of field-growth concord grapevines. American Journal of Enology and Viticulture, Davis, v.45, n.2, p.252-258, 1994.

REYNOLDS, A.G.; WARDLE, D.A.; NAYLOR, A.P. Impact of training system, vine spacing, and basal leaf removal on Rieslling. Vine performance, berry composition, canopy microclimate, and vineyard labor requirements. American Journal of Enology and Viticulture, Davis, v.47, n.1, p.63-76, 1996.

REYNOLDS, A.G.; POOL, R.M.; MATTICK, L.R. Influence of cluster exposure on fruit composition and wine quality of Seyval blanc grapes. Vitis, Landau, v.25, p.85-95. 1986.

RIBEREAU-GAYON, P. The anthocyanins of grapes and wines. In: MARKAKIS, P. (Ed.) Anthocyanins as Food Colors. New York: Academic Press, 1982. p.209-244.

RIBÉREAU-GAYON， P.; Dubourdieu,D.; Donèche, B.; Lonvaud, A. Traité d'enologie.
1. Microbiologie du vin-Vinifications.

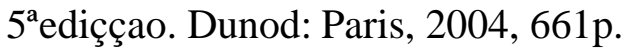

RIZZON, L. A.; MIELE, A. Avaliação da cv. Merlot para elaboração de vinho tinto. Ciência e Tecnologia de Alimentos, Campinas, 23(Supl): 156-161, dez. 2003.

SILVA, T. G.; REGINA, M. A.; ROSIER, J. P.; RIZZON, L. A.; CHALFUN, N. N. J. Diagnóstico vinícola do sul de Minas Gerais I. Caracterização físico-química dos vinhos. Ciência e Agrotecnologia, Lavras, v.23, v.3, p.632-637, jul./set., 1999.

SMART, R.E. Influence of light on composition abd quality of grapes. Acta Horticulturae, n.206, p.37-43, 1987.

SOMERS, T.C. Pigment development during ripenig of the grape. Vitis, v.14, p.269-277, 1976.

SOUSA, J.S.I. DE; MARTINS, F.P. Viticultura brasileira: principais variedades e suas características. Piracicaba: FEALQ, 2002. v.9, 368p.

SOUZA LEÃO, P. C.; SOARES, J. M. (Eds.). A viticultura no semi-árido brasileiro Petrolina: EMBRAPA. 2010. p.19-44.

Van LEEUWEN, C.; FRIANT, P.; CHONÉ, X.; TREGOAT, O.; KOUNDOURAS, S.; DUBOURDIEU, D. Influence of Climate, Soil, and Cultivar on Terroir. American Journal of Viticulture and Enology, v.55, n.3, p.207-217, 2004.

VASCONCELOS, M.C.; CASTAGNOLI, S. Leaf Canopy Structure and Vine Performance. Am. J. Enol. Vitic., v.51, n.4, p.390-396pp, 2000.

ZOECKLEIN, B.W.; JASINSKI, Y.; Mc MAHON, H. Effect of fermentation, aging, and aging sur lie on total and phenol-free Riesling glycosides. Journal of Food Composition and Analysis, v.11, n.3, p. 240248, 1998. 
ZOECKLEIN, B.W.; WOLF, T.J.; DUNCAN, N.W.; JUDGE, J.M.; COOK, M.K. Effects of fruit zone leaf removal on yield, fruit composition and fruit rot incidence of Chardonnay and white Riesling (Vitis vinifera L.) grapes. Am. J. Enol. Vitic., v.43, p.139-148, 1992.

ZUFFEREY, V.; MURISIER, F. Photosynthèse des feuilles de vigne (cv. Chasselas). I. Influence de la lumière et de la température. Revue Suisse Vitic. Arboric.

Hortic., v.32, n.6, p.341-346, 2000. 